INTERNATIONAL DESIGN CONFERENCE - DESIGN 2018

https://doi.org/10.21278/idc.2018.0364

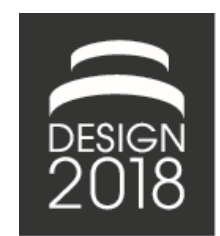

\title{
DESIGN FOR ADDITIVE MANUFACTURING: MAPPING OF PRODUCT FUNCTIONS
}

\author{
F. Valjak, N. Bojčetić and M. Lukić
}

\begin{abstract}
In this paper, authors present a new approach for supporting design process for additive manufacturing. The purpose of the method is to support structured process for the conceptual design phase and help designers utilise the unique possibilities of additive manufacturing with mapping of product functions and design principles through additive manufacturing ontology. The method is still in early research phase and therefore lacks a necessary computational framework. The paper presents the preliminary validation of the method through a case study.
\end{abstract}

Keywords: additive manufacturing, design methods, functional modelling, conceptual design, ontology

\section{Introduction}

Additive manufacturing (AM) through its characteristic of adding material precisely where it is needed, brought new design freedom and unique possibilities in design and manufacturing of various products. Unique possibilities are manifested through geometrical, material, hierarchical and functional complexity of a product (Gibson et al., 2015). AM, therefore, enables new opportunities in product design that changes the way we make products, but also the types of products we make, and thus requires new approaches to product design process that will utilise the possibilities of AM (Seepersad, 2014). This is manifested through the development of various methods and tools in the area of Design for Additive Manufacturing (DfAM). Existing DfAM methods and tools offer different possibilities in utilisation of AM in different stages of design process, especially in the embodiment and detail design. On the other hand, DfAM methods provide little or no assistance in early design phases, especially in the conceptual phase. Therefore, in this paper, a new DfAM approach is presented that will enable utilisation of AM related knowledge in the conceptual design phase by mapping of product functions with design principles for AM. The aims of the method are: to ensure a structured conceptualisation process, to help designers in finding possible solutions for parts to be produced by AM and to stimulate creativity in early design phases. Consequently, the method will help designers create new and innovative products that will utilise AM possibilities to achieve the desired functionality, integrated functions, better performances of products, and new features in products that are impossible or hardly feasible with conventional manufacturing technologies.

The structure of this paper is as follows: background and related work on DfAM methods and tools are found in Section 2, while problems of using AM in the conceptual design phase, together with existing DfAM methods for the conceptual design, are explained in Section 3. The method and research methodology are presented in Section 4, and the preliminary results gathered through a case study are presented in Section 5. The method results are discussed in Section 6. The paper concludes with Section 7. The main purpose of this paper is presenting a new approach in DfAM for conceptual design. The 
findings presented in this paper are still in early phase of research and are part of ongoing doctoral research performed by the first author.

\section{Background and related work}

Traditional design methods are developed to support conventional manufacturing processes and are thus limited in the design process with constraints imposed by manufacturing processes for which they are developed. As additive manufacturing brought new possibilities in manufacturing compared to conventional manufacturing processes, and at the same time imposed different constraints, traditional design methods proved inadequate for the development of products that will be manufactured with AM. Additionally, in recent years additive manufacturing evolved from rapid prototyping process to standard manufacturing process (Gibson et al., 2015), and thus a need for DfAM methods and tools to support design process in all stages emerged. Researchers approached this problem by investigating design potentials of AM and its manufacturing limitations, but also with the development of methods for helping designers in creating AM-conformal designs (Kumke et al., 2016).

\subsection{DfAM guidelines}

Development of DfAM started with establishing various AM design rules and guidelines based on the possibilities and limitations of AM that arise from the principle of adding material to the workpiece, type of processed material and technical capabilities of AM machines (Meisel and Williams, 2015). Therefore, the early research in DfAM was focused on determining limitations of AM to establish basic design rules and ensure the manufacturability of AM parts. This provided a basis for the development of various design guidelines for DfAM that can be divided into two types of guidelines: general design guidelines and specific guidelines. General design guidelines are focused on encouraging designers to use generic possibilities of AM in their designs like complex geometry, functional integration, part consolidation, or topology optimisation (Rosen, 2014) without proper instructions how to utilise those possibilities. At the same time, general guidelines note the need for abandoning design constraints set by conventional manufacturing processes and embracing of AM advantages (Becker et al., 2005). On the other hand, specific guidelines are usually designed for only one AM process, and therefore they lack the universal approach. Their focus is on ensuring manufacturability of a product with instructions about minimum wall thickness, maximum overhanging distances, material removal, support, etc. (Booth et al., 2016). Both approaches require initial design input, and for this reason, they are more useful in later design phases of embodiment and detail design rather than conceptual design. The primary focus of DfAM guidelines is therefore on the redesign of existing products and their optimisation for AM while ensuring the successful manufacturing.

\subsection{DfAM methodologies}

To defer from just redesign of existing products and enable the design of AM-conformal products researchers started developing various DfAM methodologies. The aim of DfAM methodologies is not just ensuring manufacturability, but more importantly utilisation of AM possibilities to improve overall functionality and performances of a product. The DfAM methodologies are often based on existing design methods, especially on Design for Manufacturing (DfM) and Design for Assembly (DfA) methods and their adjustment for AM. Rosen (2007) proposed a DfAM method that supports part and specification modelling in which manufacturable elements are used to extend the design space, and thus utilise the AM possibility of complex geometry forms and topology optimisation to improve the performance of a part. The method focuses on utilisation of geometrical complexity and mostly neglects other AM possibilities. In a similar way, Tang et al. (2014) proposed a method to integrate multi-level and multi-discipline design methods into a single process. They use an initial design input to generate design space in which topology optimisation on macro-level and lattice structure on meso-level are applied sequentially to fulfil the design requirements and improve the performances of a part. Both approaches are oriented on detail design and are not suitable for utilisation of AM in early design phases. Ponche et al. (2012), on the other hand, developed DfAM methodology based on the global approach to the design process for development of AM products to enable utilisation of AM possibilities earlier in 
the design process. Their method is based on functional surfaces and functional volumes that are fabricated with AM and are strongly dependent on the process characteristics as well as functional requirements. The methodology incorporates the knowledge about AM into the design and ensures the manufacturability of a product. Even though the knowledge about AM is used in early stages of the design process, the method is better suited for geometrical design because it does not incorporate any new functionalities enabled with AM into a product and does not support the conceptual design phase. To improve the functionality of AM products and support function integration Boyard et al. (2013) modified existing DfM and DfA methods for AM oriented design process to create their DfAM method. The main characteristic of the method is usage of a functional graph in the conceptual phase and parallel application of DfM and DfA methods optimised for AM. This approach enables users to recognise the functions of the product and apply knowledge and possibilities of AM in the conceptual phase, but the method is not developed to the level needed for innovations in AM design. On the other hand, Yang and Zhao (2015) developed a DfAM method that analyses the initial input, usually in the form of CAD model, to perform part consolidation on a functional level according to functional requirements to achieve function integration. In the next step, they perform structure optimisation to utilise the AM possibilities further and ensure the manufacturability of a product.

\subsection{Limitations of DfAM method}

Presented DfAM methods have different aims, while some are focused on detail design, other offer more possibilities in early design phases through functional analysis and integration, but neither method is entirely suitable for conceptual design. Furthermore, DfAM methods tend to focus only on some stages of the design process and lack integration into the common framework. To overcome the latter Kumke et al. (2016) proposed DfAM framework based on VDI 2221 design process. Their framework supports designers through entire AM design process, from planning and task clarification, through conceptual and embodiment design, to detail design phase. Through the modular design of the framework, it enables integration of different AM specific modules, but also conventional design methods. The proposed framework provides a solid base for development and integration of various modules that can be used in an appropriate design phase, and for specific AM process or even a particular AM machine. Although the hitherto developed DfAM methods and guidelines offer different possibilities in supporting AM oriented design process and can improve product functionality and performances, as well as ensure its manufacturability, neither proved to be suitable for supporting conceptual design for AM products. Consequently, the need for design methods and tools to support early design phases for development of true AM products still exists.

\section{AM related problems in the conceptual phase}

The conceptualisation is defined as "the process of creating something previously unknown or unseen: a new product" (Andreasen et al., 2015), and it is a core activity of the conceptual phase inside the design process. As AM brought new and unique possibilities in manufacturing, and those possibilities are changing the way we conceptualise products. Consequently, AM possibilities can improve design practice and enable the creation of new products. Furthermore, as the conceptual phase is commonly considered the most important design phase where designers shape the product configuration and most decisions regarding final product specification are made (Ye and Campbell, 2006), AM possibilities can have a great influence on conceptual design. The core problem of conceptualisation, especially with experienced designers, is the tendency of limiting the creativity with cognitive barriers that are based on previous experience of manufacturing limitations and constraints. AM removed most of the manufacturing constraints such as the need for tooling and avoiding overhangs. AM promises more freedom to designers, but this freedom comes with the cost of new limitations like building volume, material properties, anisotropy or building time. Another problem of utilisation of AM possibilities in conceptual phase is the lack of understanding how AM works, what the possibilities of AM are and what are the design principles of AM. To overcome these problems Bin Maidin et al. (2012) proposed AM design feature database as a tool to support the process of conceptualisation. The database is based on taxonomy, where four top-level taxons are: user fit requirements, improve functionality requirements, parts consolidation requirements, and aesthetics requirements. The 106 design features 
are categorised into those four categories. The database is interactive, and design features can be found either through question-based approach, a search function or with browsing the database. The design features in the database are presented with short description and visualisation. The research conducted on student and expert user studies showed improvement of incorporating AM possibilities into the design when the database is used for inspiration, gathering ideas and suggesting AM features in conceptualisation process. Although the tool proved to be useful in the conceptual phase, it has some drawbacks. The tool can guide the designer in search of suitable AM design features to solve the problems of conceptual design, but the tool does not support the interpretation of product functions and the links between functions and design features. This process is strongly user dependent and relies on user experience in interpreting product functions and what are appropriate design features to fulfil those functions.

Blösch-Paidosh and Shea (2017) proposed a similar approach based on Design Heuristics for AM to help designers in creating high-level concepts needed in the conceptual phase. By examining various existing AM products and artefacts, they formed a list of twenty-nine design heuristics, such as Customise geometry to user case or Embed functional material. The design heuristics offer process independent approach needed to create high-level concepts. The initial verification of the method showed usefulness and applicability in early design stages, especially in concept generation during conceptual phase. On the other hand, application and interpretation of design heuristics are user dependent and the method does not offer the structured guidance through the conceptual phase.

The both described approaches offer utilisation of AM in early design stages like no other DfAM method hitherto developed. However, they are user dependent in the interpretation of AM knowledge and incorporating AM possibilities into the design during conceptual phase. Consequently, the success of the conceptual phase depends on the experience of the designer. Furthermore, neither method offers a structured design process that will guide the designer in a step-by-step approach and enable broadening search of possible principal solutions for partial functions of a product.

\section{Method}

Conceptual design is a phase of the design process where through the structured process (Figure 1) that starts with the abstract identification of a problem, continues with establishing function structure and search for working principles, and ends with a concept that specifies principle solution of a problem (Pahl et al., 2007). A structured approach to conceptual design provides a step-by-step procedure to guide designers through the design process, thus supports a common understanding of a problem inside the design team, encourages the gathering of different ideas, and provides a mechanism for integrating partial solutions into a common working structure (Ulrich and Eppinger, 2003).

To support design process for development of AM products, and utilise the benefits of the structured process, our approach in DfAM is based on Pahl and Beitz structured conceptual design process that was already used in the DfAM (Rosen, 2007). Our approach will trough mapping of product functions with design principles for AM, enable the creation of principle solution of the given problem by utilising potentials of AM. The main purpose of the method is to support early phases of AM oriented design process and enable conceptualisation of new and innovative AM products. In this context, an AM product is not only a product made with AM technology, but a product that incorporates the unique design features and manufacturing possibilities of AM to achieve innovation, desired functionality, and better product performance compared to products manufactured with conventional manufacturing technologies. Furthermore, the method should guide designers through the structured step-by-step design process for developing AM products and help designers in finding possible solutions for product functions, suggest ideas that they would not instinctively think off, and enhance and stimulate their creativity in finding innovative solutions. Additionally, the method should help remove cognitive barriers to creativity, but also help designers overcome the subconscious limitations in design process imposed by the conventional manufacturing technologies. Furthermore, the methods should offer a universal approach to the design process of AM products that is AM process independent. 

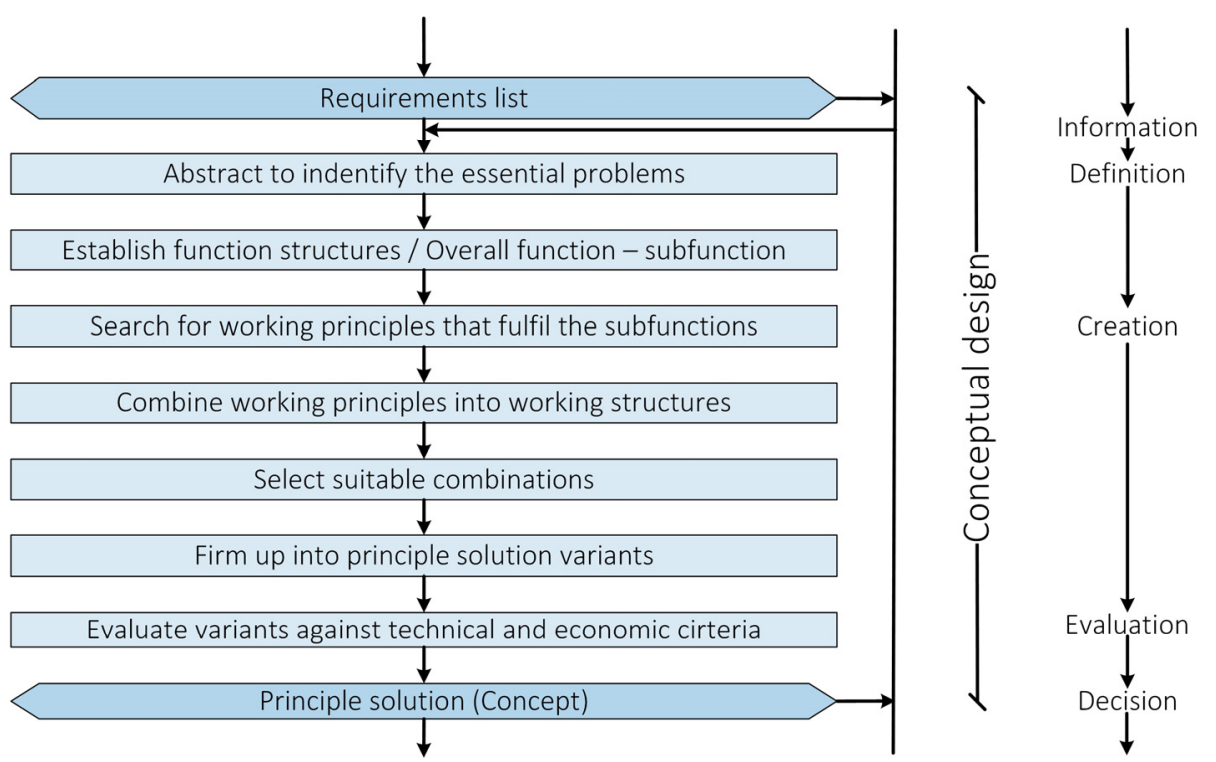

Figure 1. Steps of conceptual design (Pahl et al., 2007)

The method framework consists of four steps (Figure 2): functional decomposition, mapping of functions and AM design principles, combining of AM design principles into a working structure, and creating concept. Additionally, the method is performed inside the computational framework that, through modules for standard flow and functions vocabulary, a database of AM design principles, and AM ontology, supports the theoretical framework of the method. The first step is performed by the designer or the design team inside the computational framework. In this step function structure of the product is established. The second step is performed by the computational framework, and in this step mapping of product functions with design principles occurs. The third and fourth steps are again performed by the designers, and in these steps, a working structure and concept of the product are made. These are the steps where designers use knowledge about AM to create new and innovative products and utilise the possibilities offered by AM. Although the steps of the process are represented in the linear sequence (Figure 2) in reality, the process is iterative, and the steps are repeated until an adequate concept is created.

\section{Step 1: Functional decomposition}

The first step of the method is functional decomposition. In this step, based on the input in the form of user needs and requirements list, a function of a product is transformed into a function structure. In function structure, the overall function of a product is represented through a collection of sub-functions connected with flows of material, energy, and signal on which they operate (Stone and Wood, 2000). The use of function structure enables a clear understanding of each sub-function intent (Little et al., 1997) and improves the common understanding of product inside the design team. Furthermore, when the overall function is decomposed into smaller sub-functions, it is easier to find a technical solution for the given problem. Functional decomposition should be performed on a high-level of abstraction to avoid suggestions of technical solutions and placing limitations on designers' creativity. Therefore, the formal structured definitions of flows and functions will be used to ensure such high-level of abstraction in function structure. An example of formal standard vocabulary of flows and functions can be found in Hirtz et al. (2002) research where they defined three classes of flows (material, signal, and energy) and eight classes of functions (branch, channel, connect, control magnitude, convert, provision, signal and support). Each class has a clear definition, and it is subdivided into secondary and tertiary categories. The use of formal vocabulary, besides the mentioned benefits, enables repeatable and meaningful results and ensures a common understanding of a functional model inside the design team. Furthermore, the standard vocabulary limits the number of possible expressions in the function structure, thus simplifies computational framework for mapping functions, and enables easier integration of function structure with AM ontology and other modules inside the computational prototype framework. 


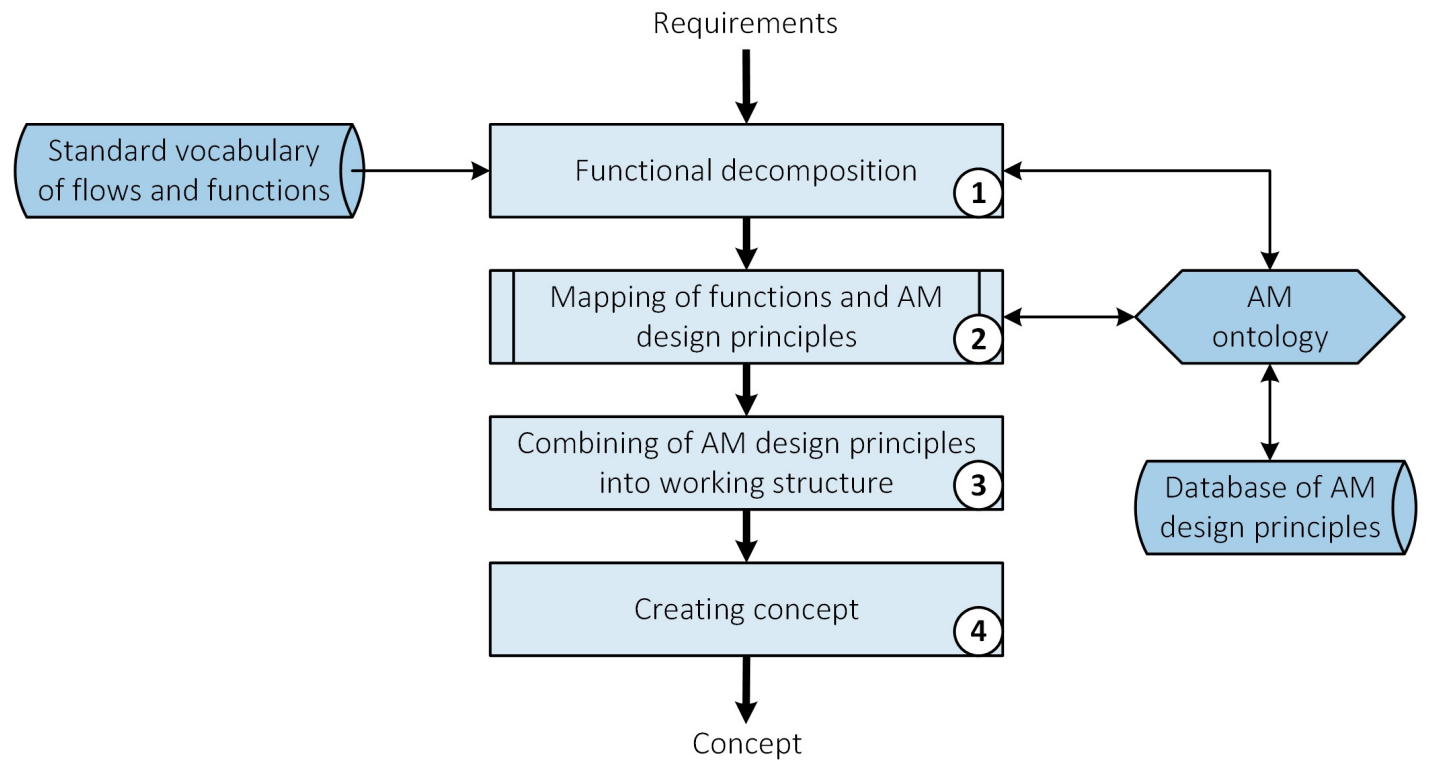

Figure 2. Mapping of functions and AM design principles

\section{Step 2: Mapping of functions and AM design principles}

The mapping of functions and AM design principles is the second step of our approach. To perform mapping firstly the function structure of the product must be analysed. The analysis of the function structure is focused on the input and output flows of each function and the sequence of functions connected by flows. Furthermore, the blocks of functions that are not connected with flows but can be solved with the same design principle needs to be identified. To perform such analysis, we propose to use AM ontology. Ontology can be defined as "an explicit specification of a conceptualisation" (Gruber, 1995) and in our approach it is used to describe a domain of AM with all its concepts and resources, to provide a common understanding of the domain (Ameri and Dutta, 2006). The purpose of the ontology is to represent the knowledge of AM domain through various classes (e.g. the AM processes, material properties, possible feature sizes, etc.) (Figure 3) and to support reasoning needed for mapping of product functions (Dinar and Rosen, 2017). We propose the use of ontology for modelling AM knowledge over a relational database as the ontologies are superior in modelling knowledge and have a richer model (expressiveness) compared to relational databases (Dinar and Rosen, 2017). Therefore, future AM ontology, through its description of the AM domain, will on its logical foundation support reasoning and through it enable analysis of the function structure needed for mapping of product functions. After performed analysis of the function structure, based on function correlation with flows and other functions inside the function structure, individual functions, as well as a block of functions, that can be solved with AM design principles will be identified and mapped with appropriate AM design principles contained in the AM design principles database. The database will contain data about various AM design principles, their description, visual representation, examples and any additional information needed for explanation of the principles to the designers. The AM design principles for the database will be formed on extensive literature review and evaluation of a predefined set of AM artefacts and products. A shape of an artefact, material and hierarchical structure, as well as other features of artefacts, will be evaluated to extract the design principles. This process will have to extract design principles on a high level of abstraction that are used as the solution for a function and are independent of other product requirements. The basis for evaluating a pool of AM artefacts and products (e.g. consumer products, medical devices, replacement parts, etc.) will include various criteria such as that the AM product incorporates unique possibilities of $\mathrm{AM}$, its performances are enhanced with $\mathrm{AM}$, or it is only manufacturable with AM. The purpose of AM design principles database is to help designers, both novices and experienced users, to understand the possibilities and design principles of AM and stimulate their creativity in the design process with descriptions, examples and visualisations of principles. The 
overall aim of this step is to offer designers different partial solutions for each sub-function of the product to enable generation of different concepts.

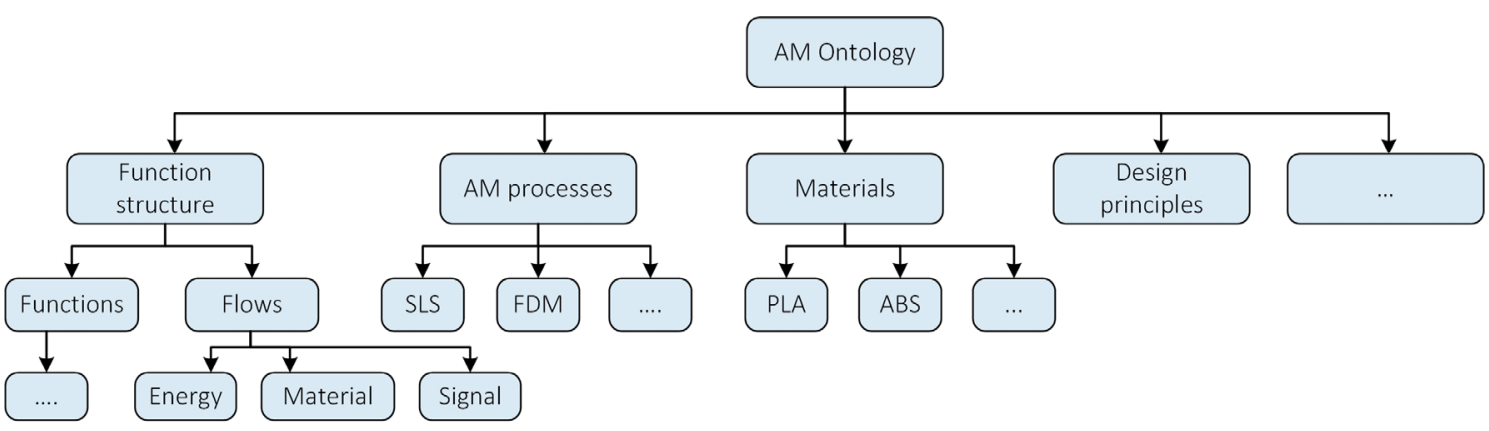

Figure 3. Part of AM ontology class structure

\section{Step 3: Combining design principles into working structure}

The mapping of functions does not create the concepts itself as this is still a cognitive process of the designer. Therefore, the next step is combining design principles from the previous step into a working structure. In this step, designer combines the principles based on different criteria, from user needs and requirements to compatibility of various design principles into a valid working structure. This step is strongly dependent on designer's skills of interpreting design principles and combining them into a working structure. For this reason, a structured process enables using of existing design tools and methods like the morphological matrix to help designers to combine the design principles.

\section{Step 4: Creating concept}

The final step of the proposed method is creating of concept. The concept is a design proposal to the given problem that shows the feasibility of a product through its detail description of a product (Andreasen et al., 2015). In our method mapping of product functions with design principles helps designers in finding solutions based on AM for the given problem. The principles itself should be used as an inspiration for the designers and to boost their creativity in creating various concepts, to fulfil the user needs and requirements. The concept creation is a cognitive process. Therefore, it depends on the designers' ability to create new and innovative solutions. The concept is described with familiar methods like hand sketching, written descriptions, CAD modelling or some other method of description. The description of a concept should be detailed enough so that the entire design team can understand the concept, and the feasibility of a concept can be evaluated.

\section{Case study}

To test and verify the feasibility of proposed method a case study was conducted. The method was used to develop a microreactor in ongoing doctoral research performed by the third author. Microreactors are process units in which at least one dimension of the channel network is between $10 \mu \mathrm{m}$ and $500 \mu \mathrm{m}$ in size (Yoshida et al., 2005), similarly to millireactors where dimensions of channel network are over 500 $\mu \mathrm{m}$. Nowadays, microreactors and millireactors are finding increasing application in many fields, from the chemical industry and biotechnology to the pharmaceutical industry and medicine. Small dimensions of the microreactor allow an extremely large surface to volume ratio, improved heat and mass transfer, shorter retention time, effective mixing, but it also allows precise regulation of the process. Microreactors offer many advantages over conventional batch scale reactors, including vast improvements in energy efficiency, reaction speed and yield, safety, reliability, scalability, on-site and on-demand production, as well as a much finer degree of process control. Research conducted hitherto have confirmed that microreactor methodology is applicable for performing gas and liquid phase reactions. They can be used for different single or multiple phase reactions and even for explosive and flammable reactions or those that use highly toxic components. Depending on the microchannel's geometry, material and physical properties of solvents, the contact between two phases can create different flow pattern. As microreactors have a small output flow, they can be connected to a series of reservoirs for chemical reagents and products to form a complete device called "chip". Transferring 
microreactors to a larger scale is achieved by adding and linking process units, so called numbering up. This enables transfer of the process from a laboratory to an industrial level. Numbering up can be performed in two ways: external numbering up (connection of many devices in parallel) and internal numbering up (parallel connection of functional elements). One of the most significant advantages of numbering up is that continuous operation is uninterrupted if one of the units fails because it can be easily replaced with no effect on other parallel units.

The microreactor is, therefore, a multifunctional product that at the same time requires small dimensions and features size, as well as a complex geometry of features. The mixing of fluids is only achieved with the static geometrical features and without an external source of energy other than the volumetric flow and pressure of the fluids themselves. As the microreactor is being developed for research purposes, additional requirements were requested, such as continuous visual monitoring of a process and fast production of prototype due to a high number of iterations. For these reasons AM was chosen for production of the microreactor and consequently for the same reasons, the microreactor was chosen for case study and preliminary validation of the proposed method for mapping product functions.

The development of the microreactor started with task clarification, continued with conceptual design in which proposed method was used, followed by embodiment and detail design and ended with manufacturing and testing of prototypes. The schema of the development process and used method can be seen in Figure 4. Before the conceptual phase started, the task was analysed, and the requirement list was created. Main requirements were: enable mixing of two liquids with static geometric features, enable visual monitoring of the process, and enable connecting of the microreactor on pumps.

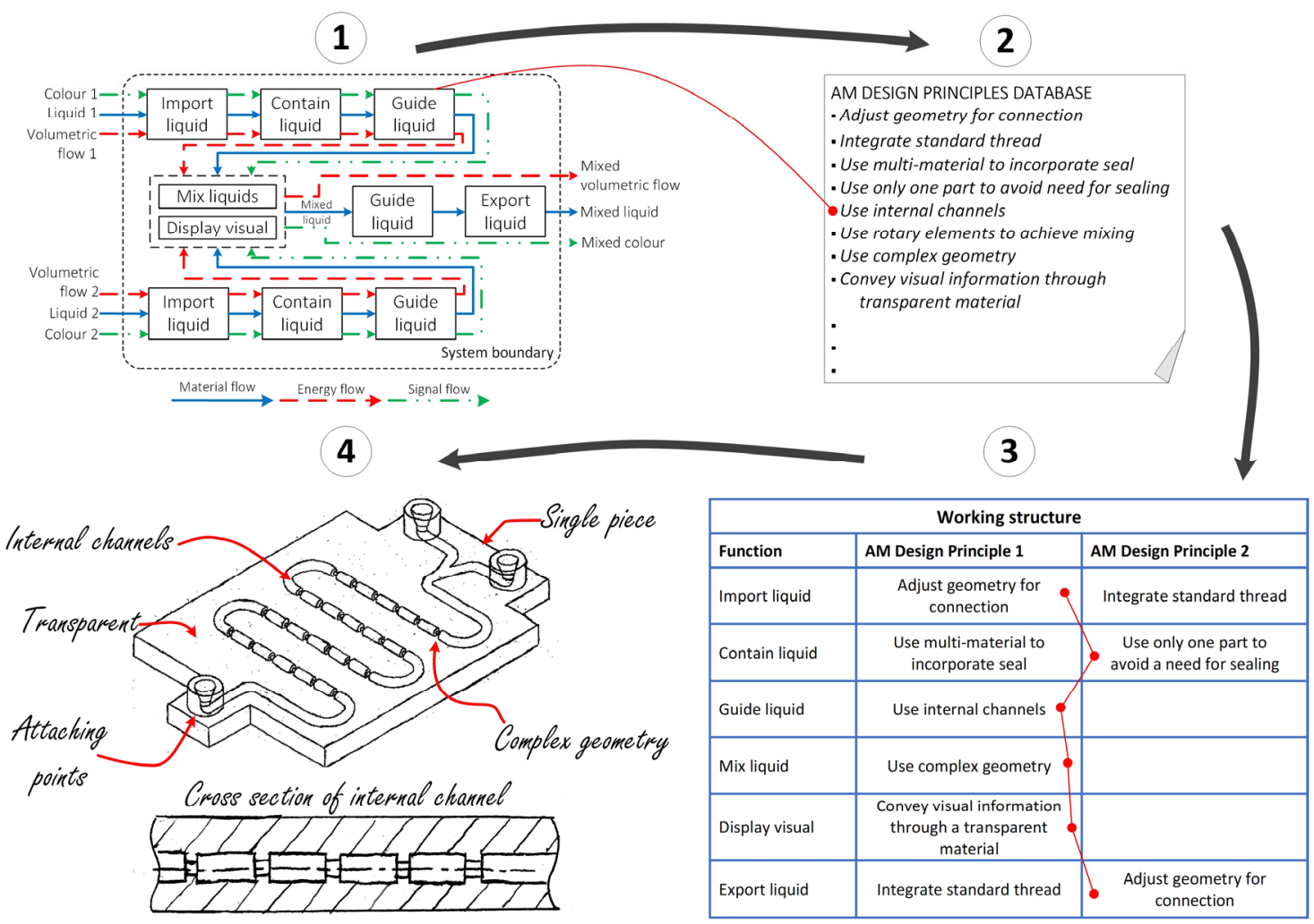

Figure 4. Schema of microreactor development through mapping of functions

In the first step of conceptual phase overall function of the microreactor was decomposed in all its subfunctions and flows. The functional decomposition was made using the vocabulary of flows and functions proposed by Hirtz et al. (2002) to achieve high-level of abstraction and avoid suggestions of any technical solutions. The function structure contains ten sub-functions interconnected with flows of energy, material and signal. After establishing function structure, in second step mapping of functions was performed. To perform mapping of functions a function structure and its sub-functions, together 
with interconnecting flows, were analysed. The focus of the analysis was on input and output flows of each function, as well as on position of each function regarding other functions and flows in the function structure. Based on the conclusions derived from the analysis, functions were mapped with possible AM design principles. For example, for function Mix liquid, as there are no entries of mechanical energy or other material in the form of a rotary element, principles such as Use rotary elements to achieve mixing are not possible, so design principle Use complex geometry is mapped. For the function Display visual there is an entry of a visual signal contained in the colour of a liquid, therefore a mapped principle is Convey visual information through a transparent material. The process of analysing and mapping of functions was performed for each function. In this case study, authors have analysed and mapped functions with the design principles as proposed computer framework is still under development. In the future, the task of analysing and identifying possible solution will be performed by AM ontology that will through its reasoner conclude, based on the input and output flows of a function and other details of function structure, which design principle are possible and suitable for each sub-function. In the third step, all design principles derived from mapping process were examined and combined into a feasible working structure. The design principles and working structure were used as the source of ideas for creating a concept of the microreactor in the final step of the method. The chosen concept of the microreactor is single piece product with internal channels that contain complex geometry in the form of various sections and obstacles to produce turbulent flow and consequently enable mixing of liquids. The microreactor is made of transparent material thus enabling continuous monitoring of the process. It also has attaching points whose geometry enables connecting of pumps with the help of chips.

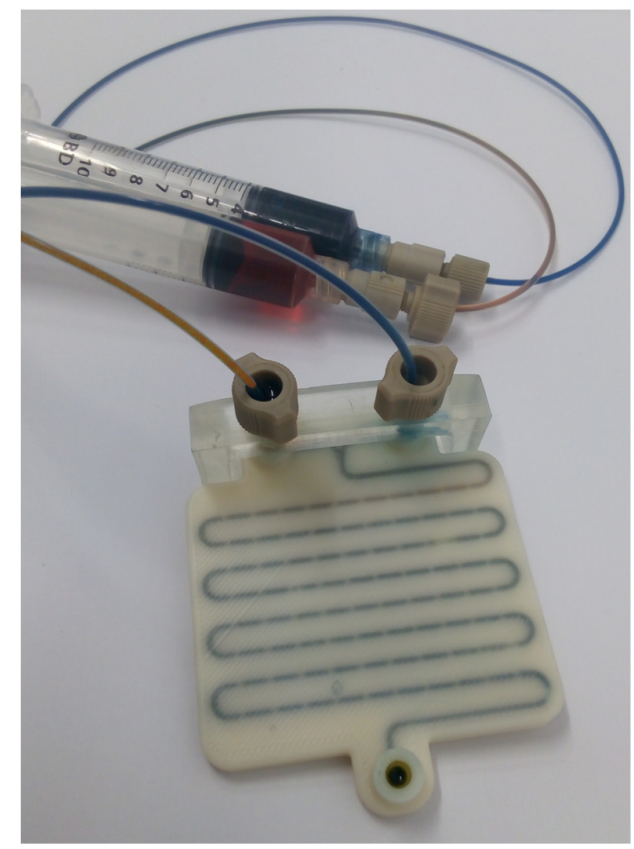

Figure 5. Prototyping and testing

To verify the feasibility of the concept created with the proposed method the development of microreactor continued with embodiment and detail design in which CAD model was created, and finished with manufacturing of the prototypes and testing. The prototypes were manufactured with two different AM process, Stereolithography (SLA) and Fused Deposition Modelling (FDM). This proved the usability of the method on multiple AM processes simultaneously, therefore demonstrating the universal approach of the proposed method. The prototype for testing presented on Figure 5 was produced on FDM desktop AM machine, from a semi-transparent ABS-like material. The testing of the prototype was performed with two liquids of different colours. As the testing equipment was connected to the microreactor's attaching point, the two liquids were pumped into the mixing channel. The change of tone at the end of a mixing channel showed the successful mixing of two liquids. The semi-transparent 
material used for manufacturing of microreactors enabled visual monitoring of the entire mixing process. The testing verified that the functional requirements stated at the beginning of development process were fulfilled.

\section{Discussion}

The presented method for conceptual design during development of AM products is ongoing research, thus the discussion is limited to the theoretical foundations of the method and the preliminary validation through the case study. The main purpose of the method is to incorporate AM knowledge, knowledge about AM possibilities, design solutions, constraints, etc., in early design phases. This is achieved through mapping of product functions with AM design principles, which enables application of AM knowledge during the conceptual phase of the design process, earlier than the majority of hitherto developed DfAM methods as their primary focus is mostly on embodiment and detail design. The conducted case study showed the applicability of the method during early design phases. The mapping of functions enabled integration of functions and supported a search of possible solutions for the given problem, which lead to the concept of the product. The purpose of the method is to support conceptual design phase. However, the output of the method is a concept with a description of applied design principles, preliminary layout, but also with details of shapes and other features of a concept. Therefore, the method is not only used for conceptual design but also for early embodiment design as well. When compared to existing DfAM methods for conceptual design (Bin Maidin and Blösch-Paidosh \& Shea) proposed method offers a systematic approach to the design process and suggests the possible AM solutions based on function structure of the designed product. Therefore, it is less dependent on the user interpretation of the possible solutions and suggest the designer solutions that he/she would not instinctively think of, thus removing the cognitive barriers to creativity. Furthermore, the method aims to offer a universal approach to conceptual phase with principles on a high level of abstraction, that are form, material and AM process independent when possible, similarly to list of heuristics from BlöschPaidosh and Shea (2017). Once developed, the method could be classified into the group of discursive design methods that offer a step-by-step approach in finding solutions, similar to Classification Schemes or Design Catalogues (Pahl et al., 2007). The discursive methods do not exclude intuition but merely guide designers and have an influence on their ability to find the possible solutions for the given problem. The discursive characteristic of the proposed method is best seen in the second step of the method, where after mapping of product functions, the method offers different possible AM design principles as partial solutions for the product sub-functions. At the same time, the proposed method acts in a similar way like other structured design methods, it offers a systematic approach through four steps of the conceptual design and therefore utilises the benefits of the step-by-step design process. In its systematic approach, the proposed method differs from other existing DfAM methods for the conceptual design phase. Additionally, the fact that the proposed method is based on the familiar design process that is widely used in practice should enable easier understanding of the method, as well as allow implementation of the method in usual design activates associated with AM design. Furthermore, the familiar framework of the method enables integration of the method in broader conventional design methods and existing DfAM methods that cover entire design process, as well as integration in possible future DfAM design process or framework.

\section{Conclusion and future work}

In this paper, a new approach for supporting design process for AM is presented. The approach intends to support early design phases by offering designers partial solutions for the given problem through mapping of product functions with design principles for AM. The proposed method offers a systematic approach through four steps. The method starts with the establishing of function structure with the predefined vocabulary that ensures modelling of function structure on high-level of abstraction without suggesting possible technical solutions. The process continues with the analysis of the function structure and mapping of functions or block of functions with the AM design principles. The design principles are then arranged in the working structure, and the concepts of products are derived from it. The preliminary validation of the method is shown through the case study. Because the method is still in early research phase the future work will be focused on (i) development of a theoretical model of the 
mapping process, (ii) development of ontology framework for mapping, (iii) development of design principles database, and (iv) validation of the method. The theoretical model would describe an analysis of functions structure, as well as establish rules for mapping of product functions. Furthermore, the ontology would enable the realisation of theoretical model and through reasoning enable mapping of product functions. On the other hand, through the development of a database, we are aiming to derive general AM design principles that are material and process independent as much as possible. In the end, the research questions and presumption would be evaluated through case and user studies, to verify the method, design principles and influence of the method on an enhancement of designers' creativity.

To conclude, the main goal of the method is to support the early phases of the design process. The method offers a structured approach to the design process and guides the designers through the conceptual and early embodiment phases. The method should help designers in creating concepts in which they will utilise unique possibilities of AM, and create new and innovative AM products, which will have better functionality and performances compared to the products designed for and manufactured with conventional manufacturing technologies. When developed, the method will be beneficial for new designers by suggesting AM design principles, as well as to experienced designers by removing cognitive barriers associated with conventional manufacturing technologies. As the method covers only part of the design process, it will have to be integrated into a broader DfAM framework to enable utilisation of AM potentials through the entire design process and ensure the manufacturability of a developed product.

\section{Acknowledgments}

This work has been supported in part by Croatian Science Foundation under the project entitled "Development of materials for 3D printing of microreactors" (UIP-2014-09-3154). The authors would like to thank Domagoj Vrsaljko and Tin Rahelić for their help during manufacturing and testing of prototypes.

\section{References}

Ameri, F. and Dutta, D. (2006), "An Upper Ontology for Manufacturing Service Description”, Proceedings of IDETC/CIE 2006 ASME 2006 International Design Engineering Technical Conferences \& Computers and Information in Engineering Conference, Philadelphia, USA, September 10-13, 2006, ASME, New York, pp. 651-661. https://doi.org/10.1115/DETC2006-99600

Andreasen, M.M., Hansen, C.T. and Cash, P. (2015), Conceptual Design: Interpretations, Mindset and Models, Springer International Publishing, Cham. https://doi.org/10.1007/978-3-319-19839-2

Becker, R., Grzesiak, A. and Henning, A. (2005), "Rethink assembly design", Assembly Automation, Vol. 25 No. 4, pp. 262-266. https://doi.org/10.1108/01445150510626370

Bin Maidin, S., Campbell, I. and Pei, E. (2012), "Development of a design feature database to support design for additive manufacturing", Assembly Automation, Vol. 32 No. 3, pp. 235-244. https://doi.org/10.1108/01445151211244375

Blösch-Paidosh, A. and Shea, K. (2017), "Design Heuristics for Additive Manufacturing", Proceedings of the 21st International Conference on Engineering Design (ICED 17) Vol 5: Design for X, Design to X, Vancouver, Canada, August 21-25, 2017, The Design Society, pp. 91-100.

Booth, J.W., Alperovich, J., Reid, T.N. and Ramani, K. (2016), "The Design for Additive Manufacturing Worksheet", Proceedings of the ASME 2016 International Design Engineering Technical Conferences \& Computers and Information in Engineering Conference IDETC/CIE 2016, Charlotte, North Carolina, USA, August 21-24, 2016, ASME, New York, p. V007T06A041; 8 pages. https://doi.org/10.1115/DETC2016-60407

Boyard, N., Rivette, M., Christmann, O. and Richir, S. (2013), “A Design Methodology For Parts Using Additive Manufacturing”, Proceedings of the 6th International Conference on Advanced Research in Virtual and Rapid Prototyping (VRAP), Leiria, Portugal, October 1-5, 2013, CRC Press, Leira, pp. 399-404. https://doi.org/10.1201/b15961-74

Dinar, M. and Rosen, D.W. (2017), "A Design for Additive Manufacturing Ontology", Journal of Computing and Information Science in Engineering, Vol. 17 No. 2, p. 21013. https://doi.org/10.1115/1.4035787

Gibson, I., Rosen, D. and Stucker, B. (2015), Additive Manufacturing Technologies: 3D Printing, Rapid Prototyping, and Direct Digital Manufacturing, Springer New York, New York. https://doi.org/10.1007/9781-4939-2113-3

Gruber, T.R. (1995), “Toward principles for the design of ontologies used for knowledge sharing?”, International Journal of Human-Computer Studies, Vol. 43 No. 5-6, pp. 907-928. https://doi.org/10.1006/ijhc.1995.1081 
Hirtz, J., Stone, R.B., McAdams, D.A., Szykman, S. and Wood, K.L. (2002), “A functional basis for engineering design: Reconciling and evolving previous efforts", Research in Engineering Design, Vol. 13 No. 2, pp. 6582. https://doi.org/10.1007/s00163-001-0008-3

Kumke, M., Watschke, H. and Vietor, T. (2016), "A new methodological framework for design for additive manufacturing", Virtual and Physical Prototyping, Vol. 11 No. 1, pp. 3-19. https://doi.org/10.1080/17452759.2016.1139377

Little, A., Wood, K. and McAdams, D. (1997), "Functional Analysis: A Fundamental Empirical Study for Reverse Engineering, Benchmarking, and Redesign”, Proceedings of DETC'97 / 1997 Design Engineering Technical Conferences, Sacramento, California, USA, September 14-17, 1997, ASME, New York, p. DETC97/DTM3879 .

Meisel, N. and Williams, C. (2015), “An Investigation of Key Design for Additive Manufacturing Constraints in Multi-Material 3D Printing”, Journal of Mechanical Design, Vol. 137 No. 11, pp. 1-9. https://doi.org/10.1115/1.4030991

Pahl, G., Beitz, W., Feldhusen, J. and Grote, K.-H. (2007), Engineering Design: A Systematic Approach, Springer London, London. https://doi.org/10.1007/978-1-84628-319-2.

Ponche, R., Hascoet, J.Y., Kerbrat, O. and Mognol, P. (2012), “A new global approach to design for additive manufacturing", Virtual and Physical Prototyping, Vol. 7 No. 2, pp. 93-105. https://doi.org/10.1080/17452759.2012.679499

Rosen, D.W. (2007), “Computer-Aided Design for Additive Manufacturing of Cellular Structures", ComputerAided Design and Applications, Vol. 4 No. 5, pp. 585-594. https://doi.org/10.1080/16864360.2007.10738493

Rosen, D.W. (2014), "What are Principles for Design for Additive Manufacturing?", Proceedings of the 1st International Conference on Progress in Additive Manufacturing Pro-AM 2017, Singapore, May 26-28, 2014, Research Publishing Services, Singapore, pp. 364-369. https://doi.org/10.3850/978-981-09-0446-3

Seepersad, C.C. (2014), "Challenges and Opportunities in Design for Additive Manufacturing”, 3D Printing and Additive Manufacturing, Vol. 1 No. 1, pp. 10-13. https://doi.org/10.1089/3dp.2013.0006

Stone, R.B. and Wood, K.L. (2000), "Development of a Functional Basis for Design", Journal of Mechanical Design, Vol. 122 No. 4, pp. 359-370. https://doi.org/10.1115/1.1289637

Tang, Y., Hascoet, J.-Y. and Zhao, Y.F. (2014), "Integration of Topological and Functional Optimization in Design for Additive Manufacturing", Proceedings of the ASME 2014 12th Biennial Conference on Engineering Systems Design and Analysis ESDA2014, Copenhagen, Denmark, June 25-27, 2014, ASME, Copenhagen, p. V001T06A006. https://doi.org/10.1115/ESDA2014-20381

Ulrich, K.T. and Eppinger, S.D. (2003), Product Design and Development, Tata McGraw-Hill Education, New York.

Yang, S. and Zhao, Y.F. (2015), “Additive manufacturing-enabled design theory and methodology: a critical review", International Journal of Advanced Manufacturing Technology, Vol. 80 No. 1-4, pp. 327-342. https://doi.org/10.1007/s00170-015-6994-5

Ye, J. and Campbell, R.I. (2006), "Supporting conceptual design with multiple VR based interfaces", Virtual and Physical Prototyping, Vol. 1 No. 3, pp. 171-181. https://doi.org/10.1080/17452750601017129

Yoshida, J., Nagaki, A., Iwasaki, T. and Suga, S. (2005), "Enhancement of Chemical Selectivity by Microreactors", Chemical Engineering \& Technology, Vol. 28 No. 3, pp. 259-266. https://doi.org/10.1002/ceat.200407127

Filip Valjak, mag. ing. mech.

University of Zagreb, Faculty of Mechanical Engineering and Naval Architecture

Chair of Design and Product Development

Ivana Lučića 5, 10000 Zagreb, Croatia

Email: filip.valjak@fssb.hr 